\title{
DIVISION XII / COMMISSION 46 / PROGRAM GROUP TEACHING FOR ASTRONOMY DEVELOPMENT
}

\author{
CO-CHAIRS \\ Edward F. Guinan, \\ Laurence A. Marschall
}

\section{ANNUAL REPORT 2008}

\section{Introduction}

Annual reports of the IAU Commission 46 Program Group Teaching for Astronomy Development (TAD) for the years 2006 and 2007 have been published in IAU Information Bulletin 100, 47; and 101, 40. Here the 2008 report is presented.

The primary goal of TAD is to aid in "the enhancement of the country's astronomy education and astronomical research in support of education." The TAD program continues to vigorously support the development of Astronomy education, teaching and research in several countries. Since the time of the IAU General Assembly in August 2006, Larry Marschall (Gettysburg College) and Edward Guinan (Villanova University) have been co-chairs of the TAD Program. TAD programs supported during 2008 include the following countries: Columbia, Kazakhstan, Mongolia, Namibia, Nepal, DPR Korea, and the Philippines. Also discussed are preliminary plans for joint International Year of Astronomy (IYA2009) and TAD programs for Africa during 2009. Summaries of the TAD sponsored programs are provided here.

\section{Kathmandu, Nepal, International School on Astronomy and Astrophysics}

An International School on Astronomy and Astrophysics (ISAA) was organized in Nepal, 28 March - 6 April 2008, by the B. P. Koirala Memorial Planetarium, Observatory and Science Museum Development Board, Ministry of Environment, Science and Technology, Nepal, jointly with the International Astronomical Union in the framework of the TAD program, Commission 46. The Government of Nepal has established the B. P. Koirala Memorial Planetarium, Observatory \& Science Museum Development Board in 1992 to establish Planetariums, Observatories and Science Museums in Nepal and conducted research activities in Astronomy, Astrophysics and Cosmology. Michèle Gerbaldi served as the IAU scientific advisor and resource person to the School.

The venue of this School follows preliminary contacts between Michle Gerbaldi and former Nepalese ISYA-participant (Thailand, ISYA-2001) Sanat Kumar Sharma who is presently Co-Executive Director of B.P. Koirala Memorial Planetarium, Observatory and Science Museum Development Board. This ISAA took place from March 28 April 6, 2008, in Kathmandu, and was honored by the presence of Dr. Catherine Cesarsky, President of the International Astronomical Union upon the invitation of the Nepali organizers. This is not the first School in Astronomy in Nepal. In 2007, a Fifth 
School on Astronomy and Astrophysics was organized (January 16-19, 2007) and targeted school teachers to give them a basic background on Astronomy \& Astrophysics. The 2008 School was focused on high school teachers (grades 11 and 12) as well as university students in Master of Science programs who have taken cosmology as one of their elective courses. A total of 32 students attended the program - all but one was from Nepal.The announcement of that School and other information can be found at the URL <www.planeta-observatory.gov.np>. Many of the participating students presented poster papers or gave oral presentations focused on their Masters of Science level research projects.

The topics covered and the lecturers are listed below:

1. Infra-red astronomy - Catherine J. Cesarsky, ESO, Germany, CEA, France

2. Stellar Evolution - Michèle Gerbaldi, Institut d'Astrophysique, France

3. Neutrino Cosmology - Udayaraj Khanal, Tribhuvan University, Nepal

4. Astro-particles - Pierre Darriulat, Institut des sciences techniques nuclaire, Vietnam

5. Exo-Planets - Edward Guinan, Villanova University, USA

6. Early Universe and Particles - M. M. Aryal, Tribhuvan University, Nepal

Practical activities and laboratory exercises were also organized by Gerbaldi and Guinan. Each participant received a set of astronomical exercises (DVD and printed leaflet) from the ESO Educational Office. These exercises have been produced by the European Space Agency (ESA) and the European Organization for Astronomical Research in the Southern Hemisphere (ESO) for use in high school.

Long term programs of scientific development have been undertaken by the Nepalese Government. The first part of this development program was the installation of a $35 \mathrm{~cm}$ telescope, which is now operational. Also, a basic course in astronomy-astrophysics is under development at the university for Master Students. It should be noted that all the teachers in grades 11 and 12 classes must have a M.Sc. degree in Physics with Cosmology/Astrophysics as their most commonly taken elective courses.

Gerbaldi and Guinan also met with a group of B.Sc. and M.Sc. students from the Tribhuvan University who had not yet graduated and were too young to participate in this ISAA according to the selection criteria. A number of the most enthusiastic of these students organized a very active Nepal Amateur Astronomy Society and have been very active organizing star parties for children and the general public. They also publish regularly astronomical (once a month) notes in one of the Kathmandu Newspapers. Also Gerbaldi, at the request of this group, gave a talk on 24 April at the NAST (National Academy of Science and Technology). Gerbaldi also had a short meeting with the Rector of NAST to discuss the training of the school teachers in the domain of Astronomy/Astrophysics via distance learning. While in Nepal, Dr. Catherine Cesarsky discussed the advantages of the future membership of Nepal in the IAU and gave advice about how Nepal can join.

\section{Mongolia}

The First International Astronomical Summer School in the People's Republic of Mongolia was held in Ulaanbaatar, 21-26 July 2008 with the support of the National University of Mongolia (NUM), which hosted the event, and the IAU-TAD program. The Summer School was the result of several years of work in advanced. John B. Hearnshaw (PG-WWDA) visited Mongolia in 2004 and presented a report on astronomy there, followed in 2006 by Mongolia's joining the IAU at the Prague General Assembly in 2006. In May 2007, TAD sponsored a visit by Dr. Katrien Kolenberg to NUM, where she presented 
an intensive 2-week course on astronomy and cemented relationships with Mongolian scientists. Plans for the summer school followed directly from Dr. Kolenberg's 2007 visit, and involved, as well as Dr. Kolenberg, an organizing committee of Prof. Lhagvajav, Prof. Batsukh G., Dr. Tsolmon R. and Mrs. Dulmaa A., all of the NUM. A series of lectures over 5 days were planned, along with an excursion to the Ulaanbaatar Astronomical Observatory at Bogd Mount. Guest lecturers included ( $i$ ) Prof. R. Jayawardhana (University of Toronto, Canada), who spoke on stars, star formation, extrasolar planets, and eclipses; ( $i i$ ) Dr. K. Kolenberg (University of Vienna, Austria), who spoke on general astronomy, variable stars, and the Sun; (iii) Prof. K. Sekiguchi (National Observatory, Japan), who spoke on extragalactic astronomy and cosmology; and (iv) Prof. George K. Miley, vice-president of the IAU (Leiden University, Netherlands), who spoke on radio astronomy.

Lectures for the first three days of the school took place in the newly-built JapanMongolia Center neighboring the buildings of the NUM, which had very modern and convenient facilities. During the last two days the lectures took place in a very nice main lecture hall the NUM. All lectures were presented with laptops and LCD projectors, which seem to be standard methods, even in the developing world. Because few attendees were at ease understanding lectures in English, lectures were spoken in English and translated by Tuguldur Sukhbold, a Mongolian student who is currently in graduate school at the University of Arizona. Details of the program and images from the Summer School can be found at <www.astromongolia2008.org>.

About 50 people registered for and attended the school. Most were students at NUM, but there were also many school teachers and amateur astronomers. In addition, three astronomers from the Democratic People's Republic of Korea attended the school: Dr Nam, Dr. Kim and Dr. Jong, the new director of the Observatory of Pyongyang. (Expenses of the North Koreans were covered by the IAU). In addition, the lectures were attended by two students, one from Austria and one from Spain, who learned about the lectures by chance while traveling through Ulaanbaatar.

The summer school was well covered in the media, including visits by four Mongolian TV stations, who interviewed organizers and lectures for broadcast. In addition, Mongolian Parliament member Mrs. Dr. Munkhtuya Budee gave an inaugural greeting. Additional activities included a formal Mongolian welcome dinner, song and dance performances, a picnic to welcome IAU vice-president Dr. Miley, a closing dinner in a Mongolian Yurt - also known as a ger - and informal dinners with local hosts.

During the visit to the National Observatory at Khureltogoot, on the slopes of the Bogd Mountain, attendees (teachers and students) were welcomed by Prof. D. Batmunkh (National Academy of Sciences), who showed the visitors the observatory and explained equipment and data acquisition with the coronagraph. In addition, there is an amateur astronomical community in Mongolia, which numbers about 60-70 mainly young people, about 25 of whom are building their own telescopes. The amateurs organized a stargazing party on the 4th night of the summer school, which was attended by about 35 people, who viewed the sky through small telescopes. The summer school ended with the awarding of certificates of attendance and an informal discussion by Dr. Miley, during which students asked general questions on astronomers and on being an astronomer.

Following the close of the formal school, the four foreign astronomers who had lectured at the summer school, along with one foreign journalist, John Bohannon, traveled to western Mongolia, where, after many adventures, they viewed the August 1, 2008 total solar eclipse under clear skies. The eclipse trip resulted in several articles, including one in Science magazine: <www.sciencemag.org/cgi/content/full/321/5894/1297b>. 
Outcomes: The school can be considered a success, as measured by the enthusiasm of attending students. When George Miley asked the students at the end of the week who wanted to become a professional astronomer, many students raised their hands. Currently, however, this would require study abroad, since only very introductory courses in astronomy are taught at the university. One of the main concerns of the students was that the salary of an astronomer is one of the lowest for Mongolian academics. Changes from higher up are needed in order to improve the professional status of astronomers in Mongolia. The support of Parliament member Dr. Mukhtuya Budee offers some cause for hope in this regard. Local astronomers work mainly in solar physics. Mongolia has excellent observing sites, comparable to those of the best professional observatories in Chile, but the only instrument regularly used is the solar coronagraph. A small telescope in a network could allow Mongolian scientists to get involved in international research projects, e.g. variable star light curves, with minimal expenditure and training. During his visit, Prof. Kaz Sekiguchi also talked to Mongolian scientists and academics about the installation of a telescope and planetarium offered by Japan. Installing larger telescopes in Mongolia presents problems with technical support; a high level of maintenance is required in a climate with such extreme temperature changes. Installing a radio telescope would also be a possibility.

Until several motivated students study abroad and bring back their knowledge, which is likely to happen in the near future, it is important to focus more attention on the teaching of astronomy in elementary school and high school. The warm response of these teachers to Dr. Miley's talks indicates that there is a very receptive audience for astronomy among Mongolian school teachers. Young astronomy teacher Dulmaa Altangerel (NUM) will organize a lecture series specifically for school teachers in November 2008 at NUM. She will base her lectures on the lecture notes and power points from the summer school.

Finally, it should be noted that Prof. Miley took advantage of the presence of the North Korean astronomers to discuss with them the status of astronomy in the DPRK, and their possible membership in the IAU. (See the report on the IAU/TAD program initiative for North Korea for more details.)

\section{Philippines}

During the last few years the TAD program has supported the travel of astronomers between Gunma Astronomical Observatory (GAO) in Japan and the visiting staff of PAGASA (Philippine Atmospheric, Geophysical, and Astronomical Administration) to give Philippine scientists experience in astronomical research and to attend lectures. Japan and GAO generously provide the training for foreign students as well as providing housing and sustenance during their visits to Japan. Several years ago Japan donated a GOTO $45 \mathrm{~cm}$ telescope to the Philippines. During March 2008 the TAD program supported the travel expenses of Dr. Hakim Malasan from nearby Indonesia to give an intensive 5 day tutorial on all aspects of modern CCD photometry to the PAGASA staff astronomers and students. The TAD program is delighted that Hakim Malasan had agreed to carry out this important work. The local contact person is Dr. Cynthia Celebre - chief, Astronomy Research and Development Section (AsRDS), PAGASA (<cynthia_celebre@hotmail.com>).

\section{Kazakhstan: initial TAD program for August 2008}

At the request of Dr. Emmanuil Ya. Vilkoviskiy, Director of Fesenkov Astrophysical Institute, Almaty, Kazakhstan, an TAD program was established to support the visit of 
Dr. Marina Romanova and Prof. Richard Lovelace (Cornell University, USA) to Kazakhstan during 9-15 August, 2008. Romanova and Lovelace visited several institutions in Almaty, gave lectures and had discussions with local scientists. Historically, Kazakhstan has a strong scientific base primarily because of previous close ties with Russia in which many scientists studied at Moscow and other Russian Universities. The main Astronomy Institute, named after Prof. V. G. Fesenkov, was founded in 1941 and is well known. In recent years a new generation of astronomers was trained by the earlier generation of scientists. In addition, some young scientists go abroad for study, work and return, thus creating an advantage to the development of Astronomy in Kazakhstan.

Recently, an Astronomy Department has been created at the Al-Farabi Kazakh National University, so that Kazakh young people can study astronomy right in Almaty. In 2008 the government of Kazakhstan decided to unite three major astronomical institutions (Fesenkov Astrophysical Institute, Ionospheric Institute and Space Research Institute) to one "National Centrum of Space Researches and Technologies" (NCSRT), which is expected to enhance Kazakhstan's Space exploration program, though the process is not easy and demands special attention to support astronomical scientific researchers. There are several small observatories around Almaty which are used for observations of different astronomical objects, for training young scholars and also to show astronomy for the public.

The visit was very successful. Romanova and Lovelace gave four lectures at the Fesenkov Institute and the Al-Farabi State University, and visited the Tjan-Shan Observatory. Prof. Lovelace gave two lectures on "Modern American Telescopes" where he described a number of telescopes under construction. Dr. Romanova gave two lectures: "Modeling of Young Stars" and "Numerical Modeling in Astrophysics" in which she concentrated on modern numerical simulations and visualization procedures in theoretical astrophysics. Also they had many discussions about mutually interesting scientific problems and possible future collaborations. Dr. Vilkoviskiy expresses his sincere thanks to the IAU for this support of Kazakhstan Astronomy.

\section{Democratic People's Republic of Korea}

Some background: While attending the 29th ISYA in Malaysia (March 2007), discussions were held with Dr. Ri Jin Yong, the science and technology Counselor at the embassy of the Democratic People's Republic of Korea (DPR Korea) in Kuala Lumpur. These discussions were lead by Michèle Gerbaldi and Ed Guinan (ISYA chair and vicechair of ISYA at that time).

Previously, Dr. Gerbaldi has established cordial contacts with Dr Yong; he has been very helpful in arranging for two young astronomers from Pyongyang Astronomical Observatory (PAO) to participate in the ISYA-2007 in Malaysia. At the ISYA, Gerbaldi and Guinan met the PAO astronomers to discuss the help that the IAU could provide to North Korean Astronomy. The PAO astronomers requested technical astronomy books and many of these have already been sent and received.

Also Dr. Karel A. van der Hucht, General Secretary of the IAU, has kindly agreed to send additional spare copies of the Cambridge University Press (CUP) IAU symposia publications and other available books to the PAO. The IAU also has agreed to add PAO to the list of libraries receiving complimentary publications from the IAU. Additional books were sent from France by Michèle Gerbaldi during 2007-08. The shipment of additional books is planned to continue. The Director of PAO, Dr. Sok Jong, extends his sincere thanks for the help provided by the IAU. 
6.1. Support for astronomers from Pyongyang Astronomical Observatory (PAO) to participate in the Pacific Rim Conference in Stellar Astrophysics (PRCSA) in

Phuket, Thailand, May 2008

As a follow-up to the cooperation between the IAU and North Korea with the Malaysia ISYA, and with the continued support of Dr. Ri Jin Yong, the TAD program sponsored two visits of PAO astronomers to regional IAU astronomy meetings during 2008. The TAD program sponsored two young astronomers from PAO to attend and participate in the 8th Pacific Rim Conference on Stellar Astrophysics (PRCSA-2008) that was held in Phuket, Thailand during 5-9 May 2008. The DPR Korea Councilor, Dr. Ri Jin Yong also attended the meeting. TAD Co-chair Ed Guinan and Dr. Yong worked together to organize the participation of the PAO astronomers. Support was provided for the DPRK astronomers to attend the conference and to meet with international astronomers and IAU representatives. This was a unique opportunity for the DPRK astronomers to meet with IAU Commission 46 program representatives including the chair of the ISYA program Dr. Jean-Pierre De Greve (Belgium), and the vice chair of the IAU Commission 46 Exchange of Astronomers Program, Dr. Kam-Ching Leung - co-organizer of the conference. Moreover, this venue was a good opportunity for the PAO astronomers to have fruitful discussions and exchanges of ideas with Dr. Boonrucksar Soonthornthun of the National Astronomical Research Institute of Thailand. The IAU appreciates the great help that Dr. Soonthornthun provided in expediting the visas for the PAO astronomers to attend the PRCSA also for generously waiving the conference registration fees for the attendees from North Korea.

The PRCSA-2008 meeting was an excellent opportunity for more extended discussions with the DPRK astronomers and government representative about the DPRK renewing its membership in the IAU and participating in IAU sponsored programs such as TAD and ISYA. As a follow-up, it was proposed that three PAO astronomers, including the new PAO Director, attend the TAD sponsored Astronomy School in Ulaanbaatar, Mongolia during late July. Dr. Karel van der Hucht and IAU Vice president Dr.George Miley kindly agreed to support this program as did the DPR- Korea and the organizers of the Astronomy School in Mongolia.

\subsection{Participation of PAO astronomers in the Mongolia Astronomy School, in July 2008}

With the continued strong support of Dr.Ri Jin Yong, the TAD program supported the participation of three PAO astronomers in the IAU-sponsored Mongolia Astronomy School held in nearby Ulaanbaatar, Mongolia. One of the main reasons for the choice of this venue was that visas are not required for the North Korean astronomers to travel to Mongolia via China. Also, the new director of PAO, Professor Jong Sok, was able to attend. In addition, PAO staff astronomers, Drs. Kim Mun-Song and Nam Sok-Chon also attended. The PAO astronomers held fruitful discussions with Mongolian astronomers and the IAU-sponsored lecturers and School organizers Drs. Katrien Kolenberg and Tsolmon Retchin.

\subsection{International collaboration}

Dr.George Miley, IAU Vice-President and Coordinator of the IAU C-46 Education Programs, attended to the School to meet with the Mongolian and PAO astronomers and students. Dr. George Miley had substantive discussions with Dr. Sok and the PAO astronomers. Three main topics were covered - $(i)$ the present situation of astronomy in the DPRK; $(i i)$ future needs and plans of the PAO and ( $i i i)$ the possibility of the DPR Korea rejoining the IAU. Also discussed were the possibilities of supporting visits from 
the DPR Korea to China. This would be a cost effective program since PAO has an agreement with NAO China in Beijing. However, the DPR Korea would need to rejoin the IAU before consideration could be given for funding of this type program.

Dr. Sok and PAO astronomers discussed other several additional aid priorities with Dr. Miley. These include: broadening the interests of the PAO into new areas, e.g., stellar astrophysics. Acquiring a small solar telescope and acquiring an adequate library of text books and access to modern journals. Also discussed was a proposal for the organization of a national astronomy school for interested physics students and school teachers, similar to the TAD school in Mongolia and hosting lectures in the DPR Korea by IAU sponsored astronomers. Dr. Miley recommended, on the longer term (next few years), the PAO should consider writing a strategic plan for the development of DPR Korea astronomy. Such a plan could help in acquiring outside funds. Dr. Miley offered to assist with the preparation of such a plan. Although help from outside for the building up DPR Korea astronomy is possible, significant internal support would also be required. Several sources of external support for the development of world astronomy require internal matching funds.

Representing North Korean Astronomy, Dr. Sok indicated that the DPR Korea astronomers wish to join the IAU as soon as possible. However, the annual dues would be a substantial burden for the PAO at this time, even those for interim membership. Dr. Sok already has had talks with the DPR Korea Academy about the matter. In that regard Dr. Sok agreed to inform the Academy in the DPR Korea about this meeting and will raise with them the matter of facilitating DPR Korea membership of the IAU as soon as possible. Also Dr. Miley agreed to explore possible European sources for funding visits by DPR Korea astronomers and investigate the possibility for future support by the IAU Commission programs.

\section{Columbia. Support for student participation in the Columbian Congress of Astronomy \& Astrophysics Meeting in Medellin}

The TAD program assisted in supporting Columbian students and to attend and participate in the Colombian Congress of Astronomy and Astrophysics (COCOA) held in Medellin, Colombia during August 2008. The purpose of the meeting was to convene for the first time professional astronomers and astronomy students from Columbia to meet with Astronomy and Astrophysics researchers from the USA, Europe and South America to exchange ideas and establish collaborations. The meeting consisted of over a dozen invited speakers, half of which were from foreign institutions, covering a broad range of topics that include star and planet formation, astronomical instrumentation, galaxy formation and solar system astronomy. The long range goal of this meeting is the development of a comprehensive roadmap for the development of sustainable Astronomy and Astrophysics education and research programs for Columbia. TAD support was requested by Juan Rafael Martinez Galarza (PhD student at Leiden Observatory). More information about the Congress can be found the web site <urania.udea.edu.co/cocoa2008/ index.php>.

\section{Islamic Republic of Iran. New initiatives}

TAD co-chair Guinan was invited to visit Iran after the 2008 ISYA in Istanbul, to discuss future cooperation with the IAU. He visited Iran from 22-29 July 2008. The invitation was extended by Dr. Yousef Sobouti, Director and President of the Institute for 
Advanced Studies in Basic Sciences (IASBS), Zanjan. During this visit Guinan represented the TAD program and exchanged ideas for developing Astronomy education and training of Iranian students. In particular he discussed areas in which the TAD program can assist. In Tehran he met with Prof. Reza Mansouri, Head of the Astronomy School at the Institute for Theoretical Physics and Mathematics (IPM: <www.ipm.ac.ir>), and also met with Drs. Habib Khososhahi and Sepehr Arbabi. They discussed the development of the Iranian National Observatory (INO) and possible assistance and advice for this project from the IAU.

Guinan visited IASBS in Zanjan for three days and met with Dr. Sobouti and other members of the institute and held discussions about the development of Astronomy education and training in Iran. Dr. Sobouti and Guinan discussed possible future IAU cooperation and assistance for astronomy education and training of Iranian students within the framework of the TAD Program. As a result of these discussions a formal proposal was made by Dr. Sobouti and the IASBS to hold a one week TAD/IAU supported Astronomy and Astrophysics School at the IASBS during the spring 2009. This School would focus on "Recent Advances in Observational Astrophysics" and is expected be attended by 35-40 students from all over Iran. This Iran Astronomy School would be aimed at advanced undergraduates and graduate MSc students as well as new $\mathrm{PhD}$ students and thus would be similar to TAD supported schools held recently in Hanoi, Nepal and Mongolia. If this school is approved, the IAU would provide partial support for outside lecturers while the IASBS would provide the support for the housing and living costs of all attendees. It is hoped that this School could be continued annually during the next 2-3 years. While at the IASBS, Guinan presented an Astrophysics seminar and also met with graduate students and post-doctoral students to give advice and to discuss research projects and career opportunities.

\section{Sub-Saharan Africa. Possible initiatives}

The TAD program this year took steps to carry out major initiatives in sub-Saharan Africa during the IYA-2009, in the spirit of the Cornerstone 11 mandate for global development of Astronomy. The TAD program advisors communicated with Kevin Govender - the Manager of the SALT Collateral Benefits Program of the South African Astronomical Observatory (Cape Town), and coordinator of various astronomy development efforts. He has established contacts in several sub-Saharan countries and serves as an advisor to the TAD program for Africa. Kevin Govender also leads a working group on Astronomy in the region (currently focused on the IYA) which has put together a plan for the development of Astronomy in Africa themed "Astronomy for Education".

Govender also has arranged for representation at the International Council for Science (ICSU) General Assembly in Maputo, Mozambique in October 2008 and leads a survey of the state of Astronomy in each of the African countries they are working with. Also he is attending the International Heliophysical Year (IHY) meeting in Nigeria during November where, among other things, he will gauge interest in organizing a sub-Saharan astronomy education meeting. At the IHY meeting he will run training workshops on basic astronomy outreach which have proven extremely popular so far (based on feedback from similar workshops in Kenya, Namibia and a number of locations across South Africa). A consolidation of astronomy education resources mainly from South Africa have already been compiled and sent to coordinators in a number of African countries.

In response to requests from Kevin Govender, and with the help of TAD co-chair Larry Marshall, the TAD arranged to have instructional astronomy materials from Project 
CLEA (Gettysburg College) sent for distribution to interested astronomy teachers at regional meetings. The TAD program also is looking forward to working closely with Dr. Peter Martinez (SAAO.) in organizing TAD programs in the region. Dr. Martinez is very knowledgeable of Astronomy in Africa and has worked over the years helping to develop programs. One plan of action being considered is an TAD sponsored one week Astronomy School to be held in the region during the IYA-2009. This school would target undergraduate Physics and Astronomy students and high school science teachers as well as Mathematics and Science outreach programs such as science centers and promotional activities by Departments of Science and Technology.

\section{North Africa and Angola. Possible initiatives}

The TAD program continues to work closely with Dr. Hassane Darhmaoui, Al Akhawayn University in Ifrane, for the development of Astronomy education, teaching and outreach programs in Morocco. During 2008 ongoing discussions and exchanges have been carried out with Dr. Khalil Chamcham (University of Oxford) with the help of Dr. Michèle Gerbaldi about a possible TAD program for Morocco. Plans for a five day TAD Astronomy School that will be primarily focused on the training of Moroccan high school science teachers (but also open to university students) are being developed for 2009.

Discussions with Dr. Roger Ferlet (IAP) have been carried out about possible help and advice from the TAD program for the establishment of the first "Ecole doctorale" in Astronomy (students who will begin PhD studies) in Algeria. Also Dr. Ferlet may be requesting IAU help for an Annual National Astronomy Festival for amateurs and others interested in Astronomy. Also in Tunisia there is an interest in working with the TAD program about the training of high school mathematics teachers in Astronomy. With French assistance, Dr. Ferlet has also expressed advice and help in the promotion of elementary Astronomy courses for high school students in Tunisia. He also reported on the establishment of an Astronomical observatory in Angola that could use some possible TAD aid and advice for related public outreach Astronomy programs in that country. Many of these programs in 2009 would be closely tied to IYA2009.

Edward F. Guinan \& Laurence A. Marschall co-chairs of the Program Group 